\title{
Induction of Labour and Scheduled Cesarean Deliveries in Twin Pregnancies at the Port-Royal Maternity Hospital in Paris France
}

\author{
Emile Papiernik, Madieh Hessabi, Corinne Dubourdieu, and Jennifer Zeitlin \\ Maternité de Port-Royal, Hôpital Cochin, Université René Descartes, Paris, France
}

\begin{abstract}
$T^{\text {he }}$ he study is a critical analysis of the decisions to induce labour or schedule cesareans in the practice of a third level referral centre, with as outcome criterion the reduction of fetal death. 783 women pregnant with twins were included from 1.1.1993 to 31.12.1998, in three groups:originally booked, referred for care during pregnancy, or transferred from another institution. The results show that an important proportion of preterm deliveries result from a medical decision to induce labour or from a scheduled cesarean in the originally booked group with even higher proportions in groups of referred and transferred women. These results are discussed in relation to fetal death rates and causes. Deaths related to fetal growth restriction were not observed in women originally booked for care. The hospital bias has been discussed. The conclusion is that decisions to minimize fetal deaths in twin pregnancies increased preterm deliveries by medical decision.
\end{abstract}

Induction of labour or scheduled cesarean deliveries are currently being employed in an increasing number of twin pregnancies before 37 weeks. These decisions increase the preterm birth rates in twin pregnancies and shape our opinion about the effectiveness of the prevention of preterm birth for twins (Tafforeau et al., 1995). This new approach has been observed in large data collections, including the data representative of all 1998 births in France (Blondel, et al., 1999) and all twin births in the United States of America from 1981 to 1998 (Kogan et al., 2000). These data collections do not describe the precise reasons for this observed increase. The best hypothesis is that the decisions to induce a preterm birth or to schedule a cesarean in a twin pregnancy before 37 weeks are intended to prevent fetal death or severe impairment related to fetal growth restriction of one or both of the twins. This cannot be measured in large population based data collections but can be tested in the practice of a large obstetrical department.

This study is a critical analysis of the decisions to induce labour or schedule cesareans in the practice of a level-three referral centre. The outcome criterion chosen here is the reduction of fetal death.

This study is intended to measure in a large practice the incidence of induced labour and of scheduled cesareans (before labour begins) and to analyse the fetal deaths then observed and their precise mechanism, whether or not related to fetal growth restriction. This will not be a demonstration of the value of this new approach, as no controlled trial was performed to test it.

A study of twin pregnancies within an examination of perinatal mortality in a population-based data collection has shown that the major causes of fetal deaths are fetal malformations, twin-twin transfusion syndrome in monochorionic twin pregnancies, and ascending infection (Chitrit et al., 1999). In an important proportion of cases, nonetheless, the cause of fetal death may be related to fetal growth restriction, which is more frequently observed in twin than singleton pregnancies and often begins early. Approximately $50 \%$ of twin babies have a birth weight below the 10th percentile of singleton birth weight at 37 weeks (Alexander et al., 1998). Uterine height is not an appropriate technique for measuring fetal growth restriction, which is recognized not from clinical signs but from repeated ultrasound scans. Its severity is assessed through Doppler velocimetry of umbilical artery and cerebral blood-flow and fetal heart-rate tracings. When signs of severity are observed, they are the reason given for the decision to induce labour or schedule a cesarean.

\section{Patients and Methods}

This study is a retrospective observation of 783 women pregnant with twins who gave birth at the Port-Royal Maternity Hospital, a level-three perinatal centre in Paris, France, from 1/1/1993 to 31/12/1998. The type of labour, that is, the manner in which it began, was recorded in a computerised file at or immediately after delivery. The three options were spontaneous labour, defined as with no medical intervention, induced delivery, defined by the presence of any intervention to induce labour, and a scheduled cesarean. Duration of gestation is estimated according to all available data, including the first-trimester ultrasound scan. This study includes all twin deliveries with a duration of gestation of at least 22 weeks.

Address for correspondence: Emile Papiernik, 123 boulevard de PortRoyal 75679 Paris France. 
A hospital population differs from a general population because of the recruitment biases related to high-risk cases. In this study, we defined three categories of patients by their antenatal care, that is, by the point in pregnancy at which they first came to our institution: The first is the group of "originally booked" women, whose antenatal care was managed from the beginning of their pregnancy by the medical team of our outpatient clinic; the second is defined as "referred" and includes the women sent to this outpatient clinic by their treating obstetrician for a consultation and thereafter followed at the clinic (at the patient's and obstetrician's request). The third group is defined as "transferred": It includes the women admitted for a complication of their pregnancy to another maternity unit and then transferred to Port-Royal.

The Paris metropolitan region has 11 million inhabitants, 160,000 births every year, and many maternity units (153 in 1996). No professional regulations govern the choice of a delivery site for twin births. Often, of course, a pregnancy complication will lead to the mother's prenatal referral or transfer to a level-three facility. Women in France may choose the maternity unit where they will give birth, and those expecting twins often seek care at a referral center that has special training in and experience with twin pregnancies and is associated with a neonatal intensive care unit (Papiernik et al., 1996). During the period of observation, there were eight level-three referral centers in the Ile de France region.

Thus, in the first group of "originally booked" twin pregnancies, some chose this hospital because it was closest to their residence, while others based their choice on its reputation among their friends and relatives. A large part of our clientele is composed of women with a previous difficult pregnancy, pregnancy loss, or fetal or neonatal death. Finally, some women came after successful infertility treatment in the nearby infertility clinic.

After analysis of the fetal and placental pathology examinations and of the clinical circumstances, one of us categorized the mechanism of fetal death into one of four major groups: Complications of monochorionic twinning, fetal infection, fetal growth restriction and fetal malformation (which includes cases in which a medical interruption of pregnancy was decided).

\section{Results}

Table 1 shows the distribution for all 783 twin deliveries according to duration of gestation and type of labour. Overall,
$56.8 \%$ of the mothers went into labour spontaneously, $24.7 \%$ were induced and $18.4 \%$ had scheduled cesareans. These figures, however, differ according to the duration of gestation: induction was relatively frequent at term, that is, at 37 weeks or more $(37 \%)$, much less frequent for a duration of gestation of 35-36 weeks (15.0\%), and rare earlier than that. The distribution of scheduled cesarean deliveries is different, with the highest proportion observed at 28/32 weeks.

Table 2 shows the type of labour according to antenatal care group (originally booked, referred or transferred). The apparent differences between these three groups for the proportion of spontaneous deliveries, induction of labour and scheduled cesareans seems to be related to their substantial differences in the distribution of the duration of gestation: The referred group had a higher proportion of preterm births than the originally booked group and the transferred group an even higher proportion of early preterm births.

In the twin births at 28/32 weeks, the proportion of scheduled cesareans was $22.9 \%$ in the early followed group, $66.6 \%$ in the referred group, and $25.6 \%$ in the transferred group. At this duration of gestation, all three groups had a low rate of medical induction of labour. Overall, an important fraction of early preterm twin births at 28/32 weeks resulted from a medical decision, most often to perform a cesarean delivery. At a duration of gestation of 33-36 weeks, the proportion of deliveries in which the physicians chose the time of birth was still higher, especially among the referred and transferred women. Birth followed induced labour, however, more often than it did cesarean delivery. For the term twin births, at 37 or more weeks, labour was induced in a high proportion of deliveries in all three groups: $46.0 \%$ of births followed induction of labour and $15.4 \%$ scheduled cesareans.

Table 3 describes the numbers and causes of fetal deaths for all twin pregnancies. The global rate was 10.2 per 1000 births (30 fetal deaths for 1566 twin births), with a rate of 5.6 per thousand for the "originally booked" group (7 fetal deaths for 1212 births), 111.2 per thousand for the referred group (12 fetal deaths for 108 births), and 104.6 per thousand for the transferred group (11 fetal deaths for 238 births). For all 1566 births in the overall twin population, only 2 deaths were due to fetal growth restriction; both occurred in the transferred group (one fetus had a birth weight of $580 \mathrm{~g}$ at 29 weeks, and the other $300 \mathrm{~g}$ at 29 weeks).

Table 1

Distribution of Spontaneous or Induced Labour or Scheduled Cesarean (Mothers) by Duration of Gestation, for All Included Twin Pregnancies Delivered at Port-Royal Maternity from 1993 to 1998.

\begin{tabular}{|c|c|c|c|c|c|c|c|c|c|c|c|c|}
\hline$\frac{\text { Gestation duration }}{\mathrm{N} \text { and }(\%)}$ & \multicolumn{2}{|c|}{ 22-27 weeks } & \multicolumn{2}{|c|}{ 28-32 weeks } & \multicolumn{2}{|c|}{ 33-34 weeks } & \multicolumn{2}{|c|}{$35-36$ weeks } & \multicolumn{2}{|c|}{37 and more weeks } & \multicolumn{2}{|c|}{ Total } \\
\hline Spontaneous & 28 & $(90.0)$ & 89 & (72.9) & 64 & $(74.4)$ & 129 & $(66.8)$ & 136 & (38.6) & 446 & (56.8) \\
\hline Induced & 0 & & 1 & $(1.4)$ & 2 & (3.2) & 29 & $(15.0)$ & 161 & $(46.0)$ & 193 & (24.7) \\
\hline \multicolumn{13}{|l|}{ Scheduled } \\
\hline Cesarean & 3 & $(9.6)$ & 32 & $(26.2)$ & 20 & $(23.3)$ & 35 & $(18.1)$ & 54 & $(15.4)$ & 144 & (18.4) \\
\hline TOTAL & 31 & $(100)$ & 122 & $(100)$ & 86 & $(100)$ & 193 & $(100)$ & 351 & $(100)$ & 783 & $(100)$ \\
\hline
\end{tabular}


Table 2

Spontaneous and Induced Labour and Scheduled Cesarean in Twin Pregnancies by Antenatal Care Group: Originally Booked (OB),Referred (Ref) or Transferred (Trans) Mothers with Twin Pregnancies, Numbers and (Percent)

\begin{tabular}{|c|c|c|c|c|c|c|c|c|c|c|}
\hline $\mathrm{N}$ and $(\%)$ & \multicolumn{2}{|c|}{$22 / 27$} & \multicolumn{2}{|c|}{$28 / 32$} & \multicolumn{2}{|c|}{$33 / 36$} & \multicolumn{2}{|c|}{$37+$} & \multicolumn{2}{|c|}{ Total } \\
\hline OB spont & 13 & & 37 & & 162 & & 125 & 337 & & \\
\hline $\mathrm{OB}$ induction & 0 & & 1 & $(2.0)$ & 20 & (7.9) & 145 & (45.9) & 166 & (27.2) \\
\hline OB sch.c.s. & 1 & (7.2) & 11 & (22.9) & 44 & (16.0) & 47 & $(14.8)$ & 103 & (16.9) \\
\hline OB all & 14 & & 49 & & 226 & & 317 & & 606 & \\
\hline Ref spont & 1 & & 2 & & 15 & & 8 & & 26 & \\
\hline Ref induction & 0 & & 0 & & 6 & (28.0) & 11 & $(49.4)$ & 17 & (19.2) \\
\hline Ref sche.c.s. & 1 & $(50.0)$ & 4 & $(66.6)$ & 5 & (23.0) & 4 & $(19.6)$ & 14 & (26.2) \\
\hline Ref all & 2 & & 6 & & 26 & & 23 & & 57 & \\
\hline Trans spont & 14 & & 50 & & 18 & & 1 & & 83 & \\
\hline Trans induct & 0 & & 0 & & 5 & (17.2) & 5 & $(56.8)$ & 10 & (9.8) \\
\hline Trans sch.c.s. & 1 & (12.9) & 17 & (25.8) & 6 & (20.7) & 3 & (33.3) & 27 & (22.7) \\
\hline Trans all & 15 & & 67 & & 29 & & 9 & & 120 & \\
\hline TOTAL & 31 & & 122 & & 281 & & 351 & & 783 & \\
\hline
\end{tabular}

Table 3

Fetal Deaths, by Antenatal Care Group: Originally Booked, Referred or Transferred Mothers of Twins by Duration of Gestation and Major Cause of Fetal Death (Complications of Monochorionic Twinning (C.monochor.), Fetal Infection, Fetal Growth Restriction (F G.restrict.), Fetal Malformation, Including Medical Interruption of Pregnancy (F.malform.))

\begin{tabular}{|c|c|c|c|c|c|}
\hline Gestation duration & 22-27 weeks & 28-32 weeks & 33-36 weeks & 37 and more weeks & Total \\
\hline \multicolumn{6}{|l|}{ Originally booked } \\
\hline C.monochor. & 1 & 0 & 1 & 0 & 2 \\
\hline Infection & 1 & 0 & 0 & 0 & 1 \\
\hline F.G.restrict. & 0 & 0 & 0 & 0 & 0 \\
\hline F.malform. & & 1 & 1 & 2 & 4 \\
\hline Total & 2 & 1 & 2 & 2 & 7 \\
\hline \multicolumn{6}{|l|}{ Referred } \\
\hline C;monochor & 1 & 0 & 2 & 0 & 3 \\
\hline Infection & 0 & 0 & 0 & 0 & 0 \\
\hline F.G.restrict. & 0 & 0 & 0 & 0 & 0 \\
\hline F.malform. & 0 & 0 & 4 & 5 & 9 \\
\hline Total & 1 & 0 & 6 & 5 & 12 \\
\hline \multicolumn{6}{|l|}{ Transferred } \\
\hline C.monochor. & 0 & 5 & 1 & 0 & 6 \\
\hline Infection & 2 & 1 & 0 & 0 & 3 \\
\hline F.G.restrict. & 0 & 2 & 0 & 0 & 2 \\
\hline \multicolumn{6}{|l|}{ F.malform. } \\
\hline Total & 2 & 8 & 1 & 0 & 11 \\
\hline GRAND TOTAL & 6 & 9 & 8 & 7 & 30 \\
\hline
\end{tabular}

No fetal death was observed among any of the twins born at 37 weeks or more, except for deaths related to severe malformations ( 2 in the originally booked group, 5 in the group of referred group, and none in the transferred groups). Malformations also accounted for most of the fetal deaths at a gestational age of 33/36 weeks ( 1 in the originally booked group, 4 in the referred group, and none in the transferred group). The complications related to monochorionic pregnancies accounted for 11 fetal deaths overall, with 2 acardiac fetuses and 9 deaths related to a twin-twin transfusion syndrome. 


\section{$\overline{\text { Discussion }}$}

This hospital population is biased because these women expecting twins chose to receive their care in this hospital for specific reasons or were referred by their obstetrician; the data thus differ substantially from population-based data. The rate of preterm birth is higher than that observed for the entire population of twin births in a defined district in the same region (around Paris) collected in 1989-1990. On the other hand, it is less certain that population-based data will accurately include all pregnancies at the lower gestational age limit (Kiely, 1998; Papiernik et al., 1996), while in hospital records, all pregnancies are included from 15 weeks onward. Hospital records also present the advantage of a more standardized definition of the medical interventions (Kogan et al., 2000). For these reasons, the results here cannot be extended to the entire population of women pregnant with twins in the Paris metropolitan region (Ile de France).

At this time, the rate of preterm births no longer reflects spontaneous preterm births, for an increasing proportion of preterm births result from a medical decision to induce labour or schedule a cesarean delivery. This phenomenon has been measured in France in data collections representative of all births in France in 1972, 1981, and 1995 (Blondel et al., 1999). In a level-three hospital population such as that of Port-Royal, the proportion of induced preterm births reached $55 \%$ of all births at 26-32 weeks for the years 1993 to 1998 (Papiernik et al., 2000). The major reason for medically decided preterm birth is the recognition of fetal growth restriction and the prevention of the death of the growth restricted fetuses (Papiernik et al., 2000). In singleton pregnancies these medically decided births appear to be effective in reducing fetal deaths. For twin pregnancies, however, available information remains sparse. Our findings are confirmed in the US national data: An increased number of twins are born preterm via induced labour or scheduled cesarean delivery (Kogan 2000). Nonetheless, most published studies on the distribution of preterm births in twin pregnancies do not reveal the proportion due to medical decision.

The observational design of the present study does not allow any conclusions to be drawn about a possible causal relation between the new policy of more medical interventions in twin pregnancies and the reduction in the number of fetal deaths related to fetal growth restriction. This result, however, conforms to the expressed aim of reducing fetal deaths and especially fetal deaths related to fetal growth restriction to the lowest possible levels: In the 1212 births to the 606 women receiving all their care from the medical team of our outpatient clinic, there were no fetal deaths due to fetal growth restriction; nor were any such deaths observed in the 108 births to referred women. The only two cases occurred among the transferred women.

\section{Conclusion}

Currently a substantial fraction of preterm births in twin pregnancies result from medical decisions to induce labour or schedule a cesarean delivery. This new approach makes it difficult to analyse the effectiveness of measures to prevent preterm births in twin pregnancies. It appears that the expressed aim of prenatal care of twin pregnancies - to reduce to the lowest possible levels the number and rate of fetal deaths related to fetal growth restriction — is being reached.

\section{$\overline{\text { References }}$}

Alexander, G. R., Kogan, M., Martin, J., \& Papiernik, E. (1998). What are the fetal growth patterns of singletons, twins and triplet in the United States. Clinical Obstetrics and Gynecology, 41(1), 115-126.

Blondel, B., Norton, J., du Mazubrun, C., \& Breart, G. (1999). Enquète Nationale Périnatale 1998 (Vol.1). Paris: Ministère de l'Emploi et de la Solidarité. Institut National de la Santé et de la Recherche Médicale.

Chitrit, Y., Filidori, M., Pons, J. C., Duyme, M., \& Papiernik, E. (1999). Perinatal mortality in twin pregnancies: A three years analysis in Seine-Saint-Denis (France).

European Journal of Obstetrics \& Gynecology and Reproductive Biology, 86, 23-28.

Kiely, J. L. (1990). The epidemiology of perinatal mortality in multiple pregnancies. Journal of Urban Health: Bulletin of the New York Academy of Medicine, 66, 618-622.

Kogan, M. D., Alexander, G. R., Kotelchuch, M., MacDorman, M. F., Buekens, P., Martin, J. A., \& Papiernik, E. (2000). Trends in twin birth outcomes and prenatal care utilization. Journal of the American Medical Association, 284, 335-341.

Papiernik, E., Goffinet, F., Grange, G., Tantau, J., \& Cabrol, D. (2000). Mechanisms of fetal death in 783 twin pregnancies from 22 weeks at a level 3 perinatal center 1993-1998: A quality analysis. Prenatal and Neonatal Medicine, 5, 349-356

Papiernik, E., Richard, A., Tafforeau, J., \& Keith, L. (1996). Social groups and prevention of preterm birth in a population of twin mothers. Journal of Perinatal Medicine, 24, 669-676.

Tafforeau, J., Papiernik, E., Richard, A., \& Pons, J. C. (1995). Is prevention of preterm births in twin pregnancies possible? Analysis of the results of a prevention program in France. European Journal of Obstetrics or Gynecology and Reproductive Biology, 59, 169-174. 